\title{
Valence bound ground states in quantum antiferromagnets and quadratic algebras
}

\author{
V. Rittenberg* \\ Physikalisches Institut, Universität Bonn, Nussallee 12, D-53115 Bonn \\ E-mail:vladimir@th.physik.uni-bonn.de
}

ABSTRACT: The wave functions corresponding to the zero-energy eigenvalue of a one-dimensional quantum chain Hamiltonian can be written in a simple way using quadratic algebras. We describe here two applications. The non-hermitian Hamiltonians related to one-dimensional stochastic processes have always zero-energy eigenvalues corresponding to the stationary states. A class of quadratic algebras corresponding to diffusion processes was classified. As a second application, for a class of q-deformed $O(N)$ symmetric antiferromagnetic quantum chains, we give the zero-energy wavefunctions for periodic boundary conditions corresponding to momentum zero and for zone boundary states. We also consider free and various non-diagonal boundary conditions. All correlation lengths are derived.

\section{Introduction}

We will bring together two objects: one in mathematics (quadratic algebras), and one in physics (quantum chains). Let us start with the first one.

A quadratic associative algebra with $\mathrm{N}$ generators $x_{\alpha}(\alpha=1,2, \ldots, N)$ is defined by $M$ relations:

$$
\sum_{\alpha, \beta=1}^{N} c_{\alpha, \beta}^{i} x_{\alpha} x_{\beta}+\sum_{\alpha=1}^{N} d_{\alpha}^{i} x_{\alpha}=0
$$

where $i=1,2, \ldots, M$. Here $c_{\alpha, \beta}^{i}$ and $d_{\alpha}^{i}$ are complex numbers [1] [-1] - Special cases of quadratic algebras are Lie algebras and superalgebras. An algebra is of PBW (Poincaré-Birkhoff-Witt) type if one can order the generators $x_{\alpha}$ (through relabeling) such that the monomials

$$
x_{1}^{m_{1}} x_{2}^{m_{2}} \ldots x_{N}^{m_{N}}
$$

form a basis in the algebra. If the algebra is of PBW type, the number of relations $M$ is related to the number of generators $\mathrm{N}[2 \overline{2}]$ :

$$
M=N(N-1) / 2
$$

*Research supported by EU TMR network no. FMRX-CT96-0012.
We now discuss quantum chains. The most general Hamiltonian for a one-dimensional system with $N$ states, $L$ sites and nearest-neighbor interactions has the following expression:

$$
H=\sum_{k=1}^{L-1} H_{k}+\mathcal{L}+\mathcal{R} .
$$

The bulk terms $(k=1, \ldots, L-1)$ and the left and right boundary terms are

$$
\begin{gathered}
H_{k}=\sum_{\alpha, \beta, \gamma, \delta=1}^{N} \Gamma_{\gamma, \delta}^{\alpha, \beta} E_{k}^{\gamma \alpha} E_{k+1}^{\delta \beta} \\
\mathcal{L}=\sum_{\alpha, \beta=1}^{N} L_{\beta}^{\alpha} E_{1}^{\beta \alpha}, \mathcal{R}=\sum_{\alpha, \beta=1}^{N} R_{\beta}^{\alpha} E_{L}^{\beta \alpha} .
\end{gathered}
$$

Here $E_{k}^{\alpha \beta}$ are a basis for $N \times N$ matrices on the $k$-th site:

$$
\left(E^{\alpha \beta}\right)_{\gamma \delta}=\delta_{\alpha \gamma} \delta_{\beta \delta} \quad(\alpha, \beta, \gamma, \delta=1, \ldots, N) .
$$

We will assume that $H$ has at least one eigenstate of energy zero

$$
H \mid 0>=0
$$

Our aim is to describe the wavefunction $\mid 0>$ in a simple way. In order to do so, we consider 
the associative algebra defined by the bulk interaction:

$$
\sum_{\alpha, \beta=1}^{N} \Gamma_{\gamma \delta}^{\alpha \beta} x_{\alpha} x_{\beta}=x_{\gamma} X_{\delta}-X_{\gamma} x_{\delta}
$$

This algebra has $2 N$ generators $x_{\alpha}$ and $X_{\alpha}(\alpha=$ $1, \ldots, N)$. One can show that the quadratic algebra (1.9) exists [4]. This is remarkable since it is not simple to get associative algebras and it is a puzzle how the relation (1.8) (for any number of sites!) knows to produce associative algebras. Consider now a Fock-like representation of the algebra (1.9) defined by the relations:

$$
\begin{array}{r}
<V_{K} \mid\left(X_{\alpha}-\sum_{\beta=1}^{N} L_{\alpha}^{\beta} x_{\beta}\right)=0 \\
\left(X_{\alpha}+\sum_{\beta=1}^{N} R_{\alpha}^{\beta} x_{\beta}\right) \mid W_{K}>=0
\end{array}
$$

Here the $<V_{K} \mid$ and $\mid W_{K}>$ are the bra and ket reference states defined by the equations (1.10) in AUXILIARY spaces. The basis in the ket vector space in which the Hamiltonian acts is:

$$
\mid \alpha_{1}, \alpha_{2}, \ldots, \alpha_{L}>\left(\alpha_{k}=1,2, \ldots, N\right)
$$

One can prove [1] 14 that the unnormalized vacuum has the expression:

$$
\begin{gathered}
\left|0>=\sum_{\substack{\alpha_{1}, \ldots, \alpha_{L}=1 \\
\mid \alpha_{1}, \alpha_{2}, \ldots \alpha_{L}>}}^{N}<V_{K}\right| x_{\alpha_{1}} \ldots x_{a_{L}} \mid W_{K}>.
\end{gathered}
$$

Notice that the generators $\left.X_{(} \alpha\right)$ don't appear in the expression of the wavefunction.. One can also show [4] that all energy-zero wavefunctions can be obtained in this way. In the case of periodic boundary conditions, and translationally invariant zero-energy eigenfunctions, one can use the expression (1.12) making the substitution:

$$
<V_{K}|\ldots| W_{K}>\rightarrow \operatorname{Tr}(\ldots)
$$

provided that the algebra (1.9) has the trace operation. Ground-state wavefunctions can correspond to zone-boundary states (momentum $\pi$ ). One can show [15] that if the algebra has the $S t r$ operation with the properties:

$$
\begin{aligned}
& \operatorname{Str}\left(x_{\alpha_{1}} x_{\alpha_{2}} \ldots x_{\alpha_{L}}\right)= \\
& \quad-\operatorname{Str}\left(x_{\alpha_{L}} x_{\alpha_{1}} x_{\alpha_{2}} \ldots x_{\alpha_{L-1}}\right) \\
& \operatorname{Str}\left(X_{\alpha_{1}} x_{\alpha_{2}} \ldots x_{\alpha_{L}}\right)= \\
& \quad-\operatorname{Str}\left(x_{\alpha_{L}} X_{\alpha_{1}} x_{\alpha_{2}} \ldots x_{\alpha_{L-1}}\right)
\end{aligned}
$$

than the ket vector

$\left|0>=\sum_{\alpha_{1}, \ldots \alpha_{L}=1}^{L} \operatorname{Str}\left(x_{\alpha_{1}} \ldots x_{\alpha_{L}}\right)\right| \alpha_{1}, \alpha_{2}, \ldots \alpha_{L}>$

is a zero-energy wavefunction of momentum $\pi$.

We are going to give now two applications. One to non-equilibrium problems and a second one to antiferromagnetic systems.

\section{2. "Diffusion" algebras}

As stressed in the introduction, in order to apply the quadratic algebras as described above, the Hamiltonian of the quantum chain has to have at least one zero-energy eigenvalue. This is always the case for stochastic processes when the Hamiltonian gives the continuous time evolution of the probability distribution. The matrices appearing in equations (1.5) and (1.6) are intensity

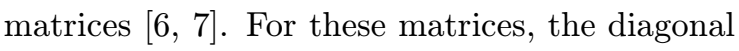
element on a given column is given by the nondiagonal matrix elements on the same column. For example:

$$
R_{\beta}^{\beta}=-\sum_{\substack{\gamma=1 \\ \gamma \neq \beta}}^{N} R_{\gamma}^{\beta}
$$

and the non-diagonal matrix elements are nonnegative numbers representing rates. For example, $R_{\beta}^{\alpha}$ is the probability that in the time interval $\mathrm{dt}$ a particle on the site $\mathrm{L}$ changes from the state $\alpha$ to the state $\beta$. The fact that the Hamiltonian has eigenvalues zero and that therefore the algebra (1.9) exists does not imply that we know to compute explicitly the matrix elements appearing in equation (1.12) or the traces respectively the supertraces in equations (1.13) or (1.15). To do that we need explicit representations. Our strategy is therefore a different one. We will consider quadratic algebras of a simpler form assuming that the generators $X_{\alpha}$ are c-numbers. We 
will look also for quadratic algebras of PBW type for which at least one representation is known (the adjoint one). Moreover we consider only processes where the $\mathrm{N}$ species of particles are conserved ("diffusion" processes). In this case, the only non-diagonal matrix elements which don't vanish are:

$$
\Gamma_{\beta \alpha}^{\alpha \beta}=g_{\alpha \beta}
$$

With these assumptions, the algebra (1.9) becomes:

$$
g_{\alpha \beta} x_{\alpha} x_{\beta}-g_{\beta \alpha} x_{\beta} x_{\alpha}=X_{\beta} x_{\alpha}-X_{\alpha} x_{\beta}
$$

where $X_{\alpha}$ 's are c-numbers. Notice that the relation (1.3) is satisfied. The question is which conditions have to be satisfied by the physical parameters $g_{\alpha \beta}$ such that the algebra (2.3) exists. Requiring the algebra to be of PBW type one obtains the equivalent of Jacobi identities:

$$
\begin{aligned}
X_{\alpha_{1}} g_{\alpha_{3} \alpha_{2}}\left(A_{\alpha_{1} \alpha_{3}}-A_{\alpha_{1} \alpha_{2}}\right) & =0 \\
X_{\alpha_{2}} g_{\alpha_{3} \alpha_{1}}\left(A_{\alpha_{2} \alpha_{3}}+A_{\alpha_{1} \alpha_{2}}\right) & =0 \\
X_{\alpha_{3}} g_{\alpha_{2} \alpha_{1}}\left(A_{\alpha_{1} \alpha_{3}}-A_{\alpha_{2} \alpha_{3}}\right) & =0(2.4) \\
X_{\alpha_{2}} X_{\alpha_{3}}\left(A_{\alpha_{2} \alpha_{3}}+g_{\alpha_{1} \alpha_{2}}-g_{\alpha_{1} \alpha_{3}}\right) & =0 \\
X_{\alpha_{1}} X_{\alpha_{3}}\left(g_{\alpha_{2} \alpha_{1}}-g_{\alpha_{3} \alpha_{2}}\right) & =0 \\
X_{\alpha_{1}} X_{\alpha_{2}}\left(A_{\alpha_{2} \alpha_{2}}+g_{\alpha_{2} \alpha_{3}}-g_{\alpha_{1} \alpha_{3}}\right) & =0
\end{aligned}
$$

where we have taken the convention:

$$
\alpha_{1}<\alpha_{2}<\alpha_{3}
$$

and have denoted

$$
A_{\alpha \beta}=g_{\alpha \beta}-g_{\beta \alpha}
$$

We call the algebras satisfying the identities (2.4), "diffusion" algebras. They have been classified ["ip]. Not much is still know about the representation theory of these algebras (see Ref. [5i] ] but the way was opened to many applications (see Ref. [i] phase transitions in stationary states. We would like to mention that in stochastic processes the zero-energy ket eigenfunctions give directly the probability distributions [i, $\left.{ }_{1}^{1}\right]$ unlike quantum mechanics.

\section{3. q-deformed $O(N)$ symmetric, N- state quantum chains}

We are going to describe a class of N-state models for which one can write the ground-states wave- functions using quadratic algebras. Unlike the Hamiltonians considered in the last section, in the present case, the Hamiltonians are hermitians. The quadratic algebras will have a very simple form: in the equations (1.9) we will be able to take all the $X_{\alpha}=0$.

Reading the paper of Reshetikhin et. al. [9i] one can notice that there are several expressions of the form (1.9) with the generators $X_{\alpha}=0$. We will choose the ones where $\Gamma_{\gamma \delta}^{\alpha \beta}$ are projector operators of rank $N(N+1) / 2-1$ for the q-deformed $B(n)$ series $(N=2 n+1)$ and $D(n)$ series $(N=2 n)$. The $x_{\alpha}$ are the generators of the non-commutative algebra of the manifold where the quantum groups act. The projector operators are going to give the bulk terms of equation (1.5). (Similar expressions for the $S p(n)$ and $O s p(m / n)$ series can also be obtained [1] $\left.\left.{ }^{\eta}\right]\right)$. We present the results for $\mathrm{N}$ even only, the case $\mathrm{N}$ odd can be

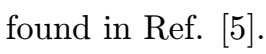

$$
\begin{aligned}
& H_{k}=\sum_{\alpha, \beta, \gamma, \delta=1}^{N} \Gamma_{\gamma \delta}^{\alpha \beta} E_{k}^{\gamma \alpha} E_{k+1}^{\delta \beta}=\frac{1}{q+q^{-1}} \\
& {\left[q \sum_{\alpha \neq \alpha^{\prime}} E_{k}^{\alpha \alpha} E_{k+1}^{\alpha \alpha}+\left(q-q^{-1}\right) \sum_{\alpha>\beta} E_{k}^{\beta \beta} E_{k+1}^{\alpha \alpha}\right.} \\
& +\delta_{N, 2 n+1} E_{k}^{\frac{N+1}{2} \frac{N+1}{2}} E_{k+1}^{\frac{N+1}{2} \frac{N+1}{2}} \\
& +q^{-1} \sum_{\alpha, \beta=1}^{N} E_{k}^{\alpha \alpha} E_{k+1}^{\beta \beta}+\sum_{\alpha \neq \beta, \beta^{\prime}} E_{k}^{\beta \alpha} E_{k+1}^{\alpha \beta} \\
& +q^{-1} \sum_{\alpha \neq \alpha^{\prime}} E_{k}^{\alpha \alpha^{\prime}} E_{k+1}^{\alpha^{\prime} \alpha} \\
& -\frac{q^{-\frac{N}{2}}}{\left[\frac{N}{2}\right]_{q}} \sum_{\alpha, \beta=1}^{N} E_{k}^{\alpha^{\prime} \beta} E_{k+1}^{\alpha \beta^{\prime}} q^{\rho_{\alpha}-\rho_{\beta}}- \\
& \left.\left(q-q^{-1}\right) \sum_{\alpha>\beta} E_{k}^{\alpha^{\prime} \beta} E_{k+1}^{\alpha \beta^{\prime}} q^{\rho_{\alpha}-\rho_{\beta}}\right]
\end{aligned}
$$

where $q$ is the deformation parameter (taken real which makes $H_{k}$ hermitian) and we use the notations:

$[n]_{q}=\frac{q^{n}-q^{-n}}{q-q^{-1}}$ and $\alpha^{\prime}=N+1-\alpha(\alpha=1, \ldots, N)$.

$\left(\rho_{1}^{\prime} \ldots, \rho_{N}\right)=(n-1, n-2, \ldots, 1,0,0,-1, \ldots,-n+1)$ 
Using (3.1) and

$$
\sum_{\alpha, \beta=1}^{N} \Gamma_{\gamma \delta}^{\alpha \beta} x_{\alpha} x_{\beta}=0
$$

with

$$
\begin{aligned}
x_{1}=a_{n}, & x_{2}=a_{n-1}, \ldots, \\
x_{n}=a_{1}, & x_{n+1}=a_{1}^{+}, \\
x_{n+2}= & a_{2}^{+}, \ldots, \quad x_{2 n}=a_{n}^{+}
\end{aligned}
$$

one finds:

$$
\begin{aligned}
& q a_{\beta} a_{\alpha}+a_{\alpha} a_{\beta}=0(\beta>\alpha) \\
& q a_{\beta} a_{\alpha}^{+}+a_{\alpha}^{+} a_{\beta}=0(\beta>\alpha) \\
& a_{\alpha}^{2}=0(\alpha=1, \ldots, n) \\
& a_{\alpha} a_{\alpha}^{+}+a_{\alpha}^{+} a_{\alpha}=q a_{\alpha+1} a_{\alpha+1}^{+}+q^{-1} a_{\alpha+1}^{+} a_{\alpha+1} \\
& \quad(1 \leq \alpha \leq n-1)
\end{aligned}
$$

The above algebra has a central element

$$
\zeta=a_{n} a_{n}^{+}+a_{n}^{+} a_{n}
$$

and an obvious representations is:

$$
\begin{aligned}
a_{k}= & 1 \otimes 1 \otimes \cdots \otimes a \otimes s^{\sigma^{z}} \sigma^{z} \otimes \\
& s^{\sigma^{z}} \sigma^{z} \otimes \cdots \otimes s^{\sigma^{z}} \sigma^{z},(k=1, \ldots, n),
\end{aligned}
$$

with

$$
a=\left(\begin{array}{ll}
0 & 1 \\
0 & 0
\end{array}\right), a^{+}=\left(\begin{array}{ll}
0 & 0 \\
1 & 0
\end{array}\right), \sigma^{z}=\left(\begin{array}{rr}
0 & 1 \\
0 & -1
\end{array}\right) .
$$

where $s=\sqrt{q}$.

In the first line of (3.8) the operator a is in the k-position. Obviously, for $q=1$ one obtains the definition of fermionic creation and annihilation operators. The algebra (3.6) has the trace operation (the representation (3.8) is finite dimensional). In order to define the Str operation, define the matrix

$$
J=\sigma^{z} \otimes \sigma^{z} \otimes \cdots \otimes \sigma^{z} .
$$

and another matrix A:

$$
A=A_{1} \otimes A_{2} \otimes \cdots \otimes A_{n} .
$$

then

$$
\operatorname{Str}(A)=\operatorname{Tr}(J A)
$$

We can now discuss the expression of the ground-state wavefunctions for various boundary conditions. For periodic boundary conditions, using (1.12) and (1.13), one finds a unique ground-state of momentum zero for $\mathrm{L}$ even and none for L odd. Similarly, using (1.16) and (3.12) one finds for $L$ even, zone-boundary states. If we consider free boundary conditions, equation (1.10) is automatically satisfied therefore the ground-state is highly degenerated and its multiplicity is simply given by the number of independent words in the algebra [i5]. If one considers various boundary conditions (diagonal or nondiagonal in respect to the $O(N)$ symmetry of the problem), one has to find which hermitian matrices $L$ and $R$ are compatible with the equations (1.10) in which we have taken $X_{\alpha=0}$ and used the representation (3.5) with (3.8). This problem is

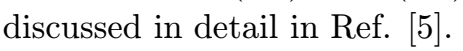

Before showing how to compute the correlation functions of the quantum chains we described, let us discuss what the latter can be good for. First let us observed that for $N=3$ and $q=1$ we recover the quantum chain of Affleck et. al. [1] i in where the expression "valence bond ground state" was used for the first time. For $N=4$ the chain can be mapped [i5] into an extended Hubbard model [1 21$]$. For all values of $\mathrm{N}$ one can map our quantum chains into various ladder models [1 13 in writing the on-rung interaction as a two-site interaction. Finally, the wavefunctions we obtain can be used as trial groundstate for more realistic models [i] $\left.1_{1}^{1}\right]$.

\section{Correlation functions}

Since the bulk Hamiltonian (3.1) is hermitian and the boundary matrices $L$ and $R$ in (1.6) are chosen hermitian, once the ket zero-energy wavefunction is known, so is the bra wavefunction (it comes from the same algebra (3.4).

Consider now two local operators $P(r)$ and $Q(s)$ acting on the sites $r$ and $s$ :

$$
\begin{aligned}
& P_{r} \mid \alpha_{1}, \alpha_{2}, \ldots, \alpha_{L}>= \\
& \quad \sum_{\beta_{r=1}}^{N} P_{\beta_{r}, \alpha_{r}} \mid \alpha_{1}, \ldots, \beta_{r}, \ldots, \alpha_{L}>
\end{aligned}
$$

$$
Q_{s} \mid \alpha_{1}, \alpha_{2}, \ldots, \alpha_{L}>=
$$




$$
\sum_{\beta_{s=1}}^{N} Q_{\beta_{s}, \alpha_{s}} \mid \alpha_{1}, \ldots, \beta_{s}, \ldots, \alpha_{L}>
$$

We want to compute the expression:

$$
G_{r, s}=\frac{<0\left|P_{r} Q_{s}\right| 0>}{Z}
$$

where $Z$ is a normalization factor coming from the fact that the wavefunctions (1.12) are unnormalized. It is useful to define the following quantities, all related to the auxiliary spaces. There are two of them, one corresponding to the ket wavefunction $(K)$ and one to the bra wavefunction $(B)$,

$$
\begin{gathered}
C=\sum_{\alpha=1}^{N} x_{\alpha} \otimes x_{\alpha} \\
P=\sum_{\alpha, \beta=1}^{N} P_{\alpha \beta} x_{\beta} \otimes x_{\alpha} \\
Q=\sum_{\alpha, \beta=1}^{N} Q_{\alpha \beta} x_{\beta} \otimes x_{\alpha}
\end{gathered}
$$

and

$$
<V_{B}\left|\otimes<V_{K}\right|=<V|;| W>=\left|W_{K}>\otimes\right| W_{B}>
$$

Using equations (1.12) and (4.2), we obtain:

$$
G_{r, s}=\frac{1}{Z}<V\left|C^{r-1} P C^{s-r-1} Q C^{L-s}\right| W>
$$

where

$$
Z=<V\left|C^{L}\right| W>
$$

For periodic boundary conditions, we have to make the following substitutions:

$$
\begin{aligned}
& <V|\ldots| W>\rightarrow \operatorname{Tr}(\ldots) \\
& <V|\ldots| W>\rightarrow \operatorname{Str}(\ldots)
\end{aligned}
$$

for translationally invariant states or for zoneboundary states, in equations (4.6) and (4.7).

Notice that $C$ plays the role of a space evolution operator in the auxiliary space but the analogy with a quantum mechanical problem can't be pushed further since $\langle V|$ and $|W\rangle$ are not eigenfunctions of $C$. Nevertheless the spectrum of $C$ gives all the correlation lengths.
The spectrum of $C$ was derived in Ref. For $N=2 n$ it is:

$$
[n-m]_{q} \quad(M=0, \ldots, N)
$$

with a degeneracy

$$
C_{N}^{N-m}
$$

Similar expressions are obtained for $N=2 n+1$. It is easy to see that for $q=1$ and large $N$, the correlation lengths become proportional to $N$ and therefore diverge in the large $N$ limit.

\section{References}

[1] G. M. Bergman, Advances in Mathematics 29(1978) 178.

[2] A. M.Vershik, Selecta Mathematica Sovietics 11(1992) 293.

[3] M. Karasev, E. Novikova, Amer. Math. Soc. Transl. bf 187 (1998) 1 and references therein.

[4] G. M.Schutz in Statistical Models, Yang-Baxter Equations and related topics, Eds. M. L. Ge and F. Y. Wu, World Scientific, Singapore, 1996; K. Krebs, S. Sandow, J. Phys. A30 (1997) 3165.

[5] F. C.Alcaraz, V. Rittenberg, J. Phys. A33 (2000) 1.

[6] B.Derrida, Physics Reports 301 (1998) 65.

[7] F. C. Alcaraz, S. Dasmahapatra, V.Rittenberg, J. Phys. A31 (1998) 845.

[8] A. P. Isaev, P. N. Pyatov, V. Rittenberg, to be published.

[9] N. Yu. Reshetikhin, L. A. Takhtadzyan, L. D. Fadeev, Leningrad Math. J. 1 (1990) 193.

[10] M. Scheunert, math QA/0004032.

[11] I. Affleck, T. Kennedy, E. H. Lieb, H. Tasaki, Commun.Math.Phys. 115 (1998) 477 and references therein.

[12] F. C. Alcaraz, R. Z. Bariev, J. Phys. A32 (1999) L483 and refernces therein.

[13] E. Dagotto, T. M. Rice, Science 271 (1996) 618. 